\title{
Comparison of Bronchoalveolar Lavage and Mini-Bronchoalveolar Lavage in the Diagnosis of Pneumonia in Immunocompromised Patients
}

\author{
M. Sezai Tasbakan ${ }^{\mathrm{a}} \quad$ Alev Gurgun ${ }^{\mathrm{a}}$ Ozen K. Basoglu ${ }^{\mathrm{a}}$ Pervin K. Ekren ${ }^{\mathrm{a}}$ \\ Husnu Pullukcu $^{\text {b Feza Bacakoglu }}{ }^{\mathrm{a}}$ \\ Departments of a Chest Diseases and ${ }^{\mathrm{b}}$ Infectious Diseases and Clinical Microbiology, Ege University School of \\ Medicine, Izmir, Turkey
}

\section{Key Words}

Bronchoalveolar lavage $\cdot$ Mini-bronchoalveolar lavage •

Pneumonia $\cdot$ Immunocompromised patient

\section{Abstract}

Background: Pneumonia is a major cause of morbidity and mortality in immunocompromised patients. Bronchoalveolar lavage (BAL) is commonly used to help diagnose and characterize pneumonia in these patients. Mini-BAL is a lessinvasive, less-costly and less-cumbersome diagnostic tool than BAL. Objectives: In this study, we compared the diagnostic value of BAL and mini-BAL in the evaluation of pneumonia in immunocompromised patients with respiratory failure. Methods: Sixty-four respiratory samples were collected from 32 immunocompromised patients admitted to our respiratory intensive care unit with a clinical diagnosis of pneumonia and respiratory failure requiring invasive mechanical ventilation. A single BAL sample and a single miniBAL sample were collected from each patient. Samples were examined for bacteriologic, mycologic, mycobacteriologic, and viral organisms. Results: The mean age of the patients was $56.0 \pm 14.4$ years. Of the $32 \mathrm{BAL}$ samples, bacterial iso- lates were detected in 11 patients (34.4\%) and on the other hand bacterial isolates were detected in 10 patients (31.3\%) of the mini-BAL samples. Fungal isolates were detected in 11 patients (34.4\%) from BAL samples and 13 patients (40.6\%) from mini-BAL samples. Our analysis demonstrated a strong positive correlation between the results of BAL and mini-BAL testing ( $r=0.850$ and $r=0.821$, respectively). Conclusion: In this study, we demonstrated a strong correlation between the isolation rates of bacteria and fungi in BAL and mini-BAL samples obtained from immunocompromised patients with pneumonia and respiratory failure. The data strongly support the use of mini-BAL sampling in such patients as a lessinvasive, less-costly and simpler alternative to traditional BAL.

Copyright $\odot 2011$ S. Karger AG, Basel

\section{Introduction}

Respiratory infection, particularly pneumonia, is a common cause of morbidity and mortality in immunocompromised patients $[1,2]$. Evidence suggests that the mortality of such patients may reach up to $50-80 \%$ if the

\section{KARGER}

Fax +4161306 1234

E-Mail karger@karger.ch

www.karger.com
(C) 2011 S. Karger AG, Basel

$0025-7931 / 11 / 0813-0229 \$ 38.00 / 0$

Accessible online at:

www.karger.com/res
M. Sezai Tasbakan

Department of Chest Diseases

Ege University School of Medicine

TR-35100 Izmir (Turkey)

Tel. +90 232390 2915, E-Mail sezai72000@yahoo.com 
diagnosis of pneumonia is accompanied by respiratory failure that requires mechanical ventilation [3]. Early, accurate diagnosis and the use of appropriate antibiotic therapy can reduce mortality rates in these patients. Diagnostic modalities including flexible bronchoscopy with bronchoalveolar lavage (BAL) and protected-specimen brush have been traditionally used to help guide the early and appropriate therapy in critically ill patients presenting with pneumonia and the effectiveness of these modalities has also been demonstrated in several studies [4-8].

Although bronchoscopic methods have high diagnostic yields, such sampling methods are costly and generally require highly experienced operators to perform them; on the other hand, the bronchoscopy itself has high risk in patients presenting with thrombocytopenia and hypoxemia. Furthermore, these invasive procedures can turn out to be complicated for reasons such as bleeding, specimen contamination, hypoxemia, airway spasm and arrhythmia [9-11].

Based on the above observations, new and less-invasive diagnostic methods such as mini-BAL and endotracheal aspiration (ETA) have been evaluated over the recent years, particularly in the diagnosis of ventilator-associated pneumonia (VAP) [12-15].

Mini-BAL was first used successfully in 1989 for the diagnosis of hospital-acquired pneumonia in a series of 59 patients [16]. Yet, there is insufficient data regarding the diagnostic value of mini-BAL and ETA compared to BAL and other bronchoscopic procedures in immunocompromised patients with pneumonia. For this reason, there is an increasing interest in the feasibility and diagnostic utility of these less-invasive methods, particularly the mini-BAL. This prospective study evaluates the diagnostic value of mini-BAL compared to BAL in immunocompromised patients with pneumonia and respiratory failure requiring mechanical ventilation. Secondarily, we compare the results of BAL and mini-BAL sampling to those of ETA.

\section{Methods}

\section{Patients}

From September 2007 to September 2009, we enrolled $32 \mathrm{im}$ munocompromised patients in this study, who were hospitalized in our respiratory intensive care unit (ICU) with bilateral pneumonia, respiratory failure, and treatment with invasive mechanical ventilation. The infiltrates were documented with chest X-ray in ICU. As the study population consisted of intubated and mechanically ventilated patients, it was impossible to perform computed tomography of the thorax. Apart from this, the long distance between the Department of Radiology and ICU was another factor.
The mini-BAL catheter is usually directed into the right lung; however, in one third of the patients it may be inserted into the left lung [13]. It was difficult to perform chest X-ray after the procedure. For these reasons, patients with bilateral pneumonia were included in the study.

Criteria for classification as 'immunocompromised' included: current presence of hematologic malignancy or solid organ tumor, a history of chemotherapy in the 6 months prior to enrollment, active long-term corticosteroid use ( $\geq 20 \mathrm{mg} /$ day prednisone equivalent for $\geq 2$ weeks), a history of high-dose corticosteroid use ( $\geq 60 \mathrm{mg}$ /day prednisone equivalent for $\geq 2$ weeks in the 3 months prior to enrollment), a history of hematopoietic stem cell or solid organ transplantation, ongoing use of cytotoxic treatment, and HIV positivity.

In the immunocompromised patients, a constellation of suggestive clinical features, a demonstrable infiltrate by chest X-rays, with or without supporting microbiological data, is considered for the diagnosis of pneumonia [17].

Isolated fungal agents from respiratory samples were considered as proven, probable or possible fungal infections due to criteria stated by the revised definitions of invasive fungal disease from the European Organization for Research and Treatment of Cancer/Invasive Fungal Infections Cooperative Group and the National Institute of Allergy and Infectious Diseases Mycoses Study Group (EORTC/MSG) Consensus Group [18].

Comprehensive analyses of patient demographics, comorbid medical conditions, types of immunocompromised states, and severity of illness by APACHE II scores were performed.

The study was approved by the local ethics committee and informed consent was obtained from the patients' relatives. On the first day at ICU, respiratory specimens were sequentially collected by ETA, mini-BAL and BAL from all patients by the same physicians (M.S.T., F.B.). The microbiological results of these samples were investigated prospectively. The samples collected by all three methods were processed by bacteriology, mycology, virology and mycobacteriology laboratories. Apart from these, CMV antigenemia was investigated in the blood samples of all patients.

\section{Mini-BAL}

Invasive mechanical ventilation $\mathrm{FiO}_{2}$ value was set to 1.0 and enteral feeding was discontinued $30 \mathrm{~min}$ prior to the procedure. In the first place, a lavage catheter (Combicath ${ }^{\mathrm{TM}}$; Plastimed, Saint-Leu-La Forêt, France) (fig. 1) was advanced into the endotracheal tube until gentle resistance was met. Then, the protective sheath at the distal end of the catheter was detached and the catheter was advanced further along the tracheobronchial tree. Radiological confirmation of catheter placement was felt to be unnecessary due to the presence of extensive bilateral infiltrates in all study subjects. Finally, $20 \mathrm{ml} 0.9 \% \mathrm{NaCl}$ was injected through the catheter and then aspirated by the same injector.

\section{Bronchoalveolar Lavage}

Following mini-BAL, $\mathrm{FiO}_{2}$ value remained at 1.0 and parameters such as oxygen saturation, pulse, or blood pressure were continuously monitored. Using a flexible bronchoscope (Olympus CLE-10, USA), we performed BAL by serial 20 -ml fractions of $0.9 \% \mathrm{NaCl}$ to a total volume of $120-150 \mathrm{ml}$. About $60 \%$ lavage volume return was assumed, an amount which we managed to obtain in our patients. 
Endotracheal Aspiration

Respiratory samples were obtained through the endotracheal tube by protected, sterile catheter method (MucoSafe ${ }^{\circledR}$; UnoplastMaersk Medical, Denmark).

\section{Microbiologic Examination}

A quantitative culture threshold of $10^{4} \mathrm{CFU} / \mathrm{ml}$ was considered significant for BAL; $10^{5} \mathrm{CFU} / \mathrm{ml}$ was considered significant for mini-BAL and ETA. The identification of bacterial isolates and antibiotic susceptibility testing were performed according to CLSI guidelines by an automated system (VITEK 2; BioMérieux Inc., Marcy-l'Etoile, France) and by conventional culture methods [19]. A mycological investigation was conducted by conventional methods. Samples were examined for fungal structures by direct microscopy and then cultivated on Sabouraud dextrose agar plates incubated at 26 and $35^{\circ} \mathrm{C}$ for 10 days. For any given study subject, a finding of $>5 \mathrm{CFU}$ on at least 2 different respiratory samples was accepted to represent fungal infection. Yeast species were identified on the basis of conventional methods, including germ tube and appearance on Tween 80 agar plate, or an automated system (ID32; BioMérieux Inc.). Samples were sent to the mycobacteriology laboratory for acid-fast bacilli smear and culture. In addition, virology tests of samples were done serologically in the virology laboratory.

\section{Statistical Analysis}

Spearman's nonparametric correlation analysis was used to assess the correlation between the various methods of respiratory sampling. Correlations were scored as weak $(\mathrm{r}=0-0.49)$, moderate $(r=0.5-0.74)$, or strong $(r=0.75-1)$. Student's t test and the $\chi^{2}$ test were used to compare the laboratory and clinical parameters of subjects in whom microbiological agents were isolated and in whom no microbiological agents were isolated, respectively $(\mathrm{p}<$ $0.05=$ significant $)$.

\section{Results}

Thirty-two immunocompromised patients with bilateral radiographic infiltrates, clinically suspected pneumonia, and respiratory failure requiring invasive mechanical ventilation were included in this study. The mean age of study subjects was $56.0 \pm 14.4$ years. Twenty-three $(71.9 \%)$ subjects were male. The demographic, clinical, and laboratory data for all subjects at the time of admission to the ICU are shown in table 1 . Of $32 \mathrm{BAL}$ samples, bacteria were isolated in $11(34.4 \%)$ and fungi were isolated in 11 (34.4\%). Of 32 mini-BAL samples, bacteria were isolated in 10 (31.3\%) and fungi were isolated in $13(40.6 \%)$ (table 2). Both bacteria and fungi were isolated in $5 \mathrm{BAL}$ samples and in $5 \mathrm{mini}$-BAL samples of the same patients. Overall, microbiological agents were detected in 17 of 32 BAL samples (53.1\%) and in 18 of 32 mini-BAL samples (56.3\%) (table 3). The correlation coefficients amongst the different sampling methods are

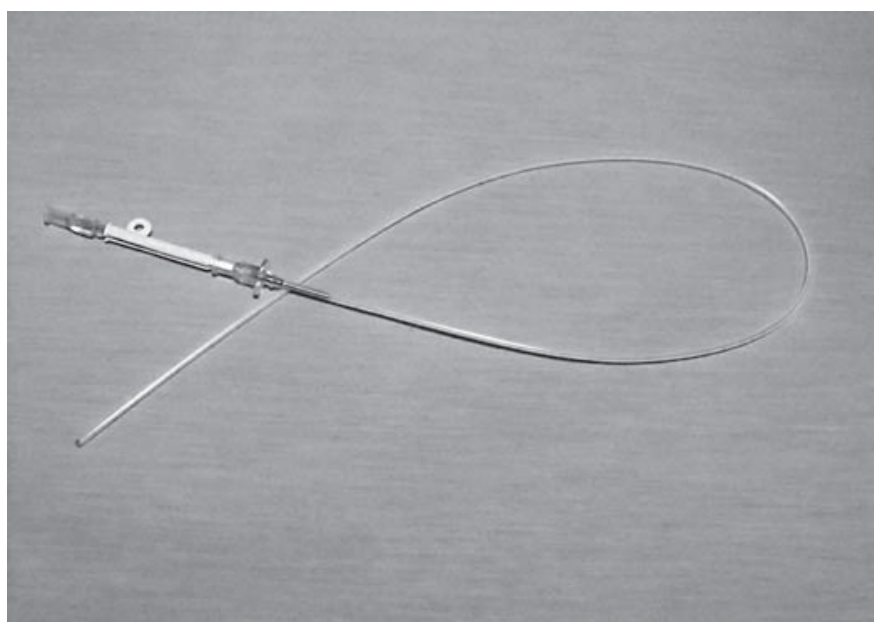

Fig. 1. Mini-BAL catheter (Combicath; Plastimed).

shown in table 4 . We demonstrated strong correlations for bacterial and fungal detection between BAL and mini-BAL $(r=0.850$ and $r=0.821$, respectively). In view of detecting bacterial agents, ETA correlated weakly both with BAL and mini-BAL $(r=0.477$ and $r=0.430$, respectively) whereas there were no correlations to detect fungal agents. Mycobacterial and viral agents were not isolated in any of the samples.

Mortality was seen in 23 subjects (71.9\%). There was no mortality difference between subjects in whom respiratory pathogens were isolated compared to subjects in whom no respiratory pathogens were isolated. There were no complications associated with any of the sampling methods.

\section{Discussion}

Our study compared the relative performance of BAL and mini-BAL for the detection of respiratory pathogens in immunocompromised patients with pneumonia and respiratory failure. We demonstrated a strong correlation between the two methods.

BAL and other bronchoscopic methods are frequently used to help diagnose pneumonia in immunocompromised patients. A prior study of 104 immunocompromised patients with pneumonia showed a microorganism isolation rate of $38 \%$ by BAL [4]. Another study of 199 patients with hematological malignancy, fever, and pulmonary infiltrates on chest imaging demonstrated an isolation rate of $59 \%$ for bacterial and/or fungal organ- 
Table 1. Demographic, clinical and laboratory data of patients on admission

\begin{tabular}{|c|c|}
\hline Age, years & $56.0 \pm 14.4$ \\
\hline Sex (male), $\mathrm{n}$ & $23(71.9 \%)$ \\
\hline \multicolumn{2}{|l|}{ Reasons for immunosuppression, $\mathrm{n}$} \\
\hline Hematological malignancy & $16(50.0 \%)$ \\
\hline Solid tumor & $10(31.3 \%)$ \\
\hline Corticosteroid treatment & $5(15.6 \%)$ \\
\hline $\mathrm{HIV}(+)$ & $1(3.1 \%)$ \\
\hline \multicolumn{2}{|l|}{ Comorbidities, $\mathrm{n}^{1}$} \\
\hline Diabetes mellitus & $13(40.6 \%)$ \\
\hline Atherosclerotic cardiac disease & $5(15.6 \%)$ \\
\hline Chronic renal failure & $5(15.6 \%)$ \\
\hline Chronic liver disease & $1(3.1 \%)$ \\
\hline No comorbidities & $11(34.4 \%)$ \\
\hline APACHE II & $23.6 \pm 7.1$ \\
\hline Sepsis, $\mathrm{n}$ & $25(78.1 \%)$ \\
\hline $\mathrm{PaO}_{2} / \mathrm{FiO}_{2}$ & $167.2 \pm 79.1$ \\
\hline Fever, ${ }^{\circ} \mathrm{C}$ & $37.0 \pm 1.1$ \\
\hline Leukocytes, $/ \mathrm{mm}^{3}$ & $12,400 \pm 17,864$ \\
\hline $\mathrm{CRP}, \mathrm{mg} / \mathrm{dl}$ & $20.8 \pm 13.2$ \\
\hline Procalcitonin, ng/dl & $4.1 \pm 5.6$ \\
\hline Albumin, g/dl & $2.7 \pm 0.6$ \\
\hline Creatinine, $\mathrm{mg} / \mathrm{dl}$ & $1.4 \pm 1.5$ \\
\hline
\end{tabular}

Values are given as number with percentage in parentheses or as mean \pm SD. APACHE II = Acute Physiology and Chronic Health Evaluation II; CRP $=$ C-reactive protein.

${ }^{1}$ Three patients have more than one comorbidity.

Table 2. Bacteriological and mycological results of BAL and mini-BAL samples

$\begin{array}{ll}\text { BAL } & \text { Mini-BAL } \\ (\mathrm{n}=32) & (\mathrm{n}=32)\end{array}$

\begin{tabular}{lcc}
\hline Bacteriological results & & \\
Acinetobacter baumannii & 4 & 4 \\
Streptococcus pneumoniae & 2 & 2 \\
MSSA & 2 & 2 \\
Cupriavidus pauculus & 1 & 1 \\
Enterococcus faecium & 1 & - \\
Pseudomonas aeruginosa & 1 & 1 \\
Yeast & 3 & 2 \\
No agent & 18 & 20 \\
Mycological results & 11 & 12 \\
Candida spp. & - & 1 \\
Aspergillus spp. & 21 & 19 \\
No agent &
\end{tabular}

Both bacteriological and mycological pathogens were detected in 5 BAL samples and in 5 mini-BAL samples from the same patients. MSSA = Methicillin-sensitive Staphylococcus aureus. isms by BAL [5]. A similar study of 93 neutropenic patients revealed respiratory microorganisms in $49 \%$ of BAL samples [6]. Yet another study demonstrated a microorganism isolation rate of $63 \%$ for BAL in a series of 57 allogeneic bone marrow transplant recipients with pneumonia [20]. Such studies support the utility of BAL in helping to characterize pneumonia pathogens in immunosuppressed patients. Our demonstration of a $53.1 \%$ isolation rate of bacterial and/or fungal pathogens by BAL in similarly immunosuppressed patients is congruent with previous findings.

Despite its high diagnostic performance in immunocompromised patients, BAL has inherent disadvantages in such patients, including the requirement for highly experienced operators and special equipment, possible complications, and high cost. However, we did not observe any complications related to the flexible bronchoscopy.

The main concern in patients with hematological malignancy is thrombocytopenia. This fact implies that BAL is not always applicable in the highest-risk patients. Although a few studies comparing bronchoscopic and nonbronchoscopic methods to evaluate immunocompromised patients with pneumonia demonstrated the diagnostic utility of both types of tests, there is a paucity of literature comparing invasive and noninvasive methods [8].

In 1989, Rouby et al. [16] used mini-BAL in the diagnosis of nosocomial pneumonia. They concluded that mini-BAL is an easily applicable, repeatable, inexpensive, and highly efficacious alternative diagnostic tool to bronchoscopic methods of respiratory sampling. Similarly, Kollef et al. [14] utilized mini-BAL in the evaluation of patients with VAP. They isolated at least one respiratory pathogen in $46.2 \%$ of cases. Another study compared the diagnostic performance characteristics of ETA and miniBAL in 82 patients with probable VAP and demonstrated that mini-BAL was significantly more specific than ETA for the microbiological diagnosis of pneumonia [21]. No patients experienced complications attributable to the mini-BAL procedure. The observations prompted the authors to conclude that mini-BAL is an effective, safe, sensitive, specific, and inexpensive tool for the serial evaluation of pneumonia in mechanically ventilated patients. Ost et al. [22] compared endotracheal aspirates, flexible bronchoscopy with protected brush or BAL, and miniBAL procedures in the diagnosis of VAP. The use of miniBAL did not improve survival but did decrease costs and antibiotic use in their patients. 
Table 3. Pathogens detected in BAL and mini-BAL samples $(\mathrm{n}=19$ patients)

\begin{tabular}{|c|c|c|c|c|c|}
\hline \multirow{2}{*}{$\begin{array}{l}\text { Age/ } \\
\text { sex }\end{array}$} & \multirow[t]{2}{*}{ Underlying condition } & \multicolumn{2}{|l|}{ Bacteriology } & \multicolumn{2}{|l|}{ Mycology } \\
\hline & & BAL & Mini-BAL & BAL & Mini-BAL \\
\hline $43 / \mathrm{F}$ & H. malignancy & A. baumannii & A. baumannii & - & - \\
\hline $54 / \mathrm{M}$ & H. malignancy & S. pneumoniae & S. pneumoniae & Candida spp. & Candida spp. \\
\hline $74 / \mathrm{M}$ & H. malignancy & - & - & - & Candida spp. \\
\hline $81 / \mathrm{F}$ & H. malignancy & Yeast & Yeast & Candida spp. & Candida spp. \\
\hline $63 / \mathrm{F}$ & H. malignancy & A. baumannii & A. baumannii & Candida spp. & Candida spp. \\
\hline $63 / \mathrm{M}$ & Solid tumor & Yeast & Yeast & Candida spp. & Candida spp. \\
\hline $45 / \mathrm{M}$ & $\mathrm{HIV}(+)$ & Yeast & - & Candida spp. & Candida spp. \\
\hline $65 / \mathrm{F}$ & Solid tumor & A. baumannii & A. baumannii & Candida spp. & Candida spp. \\
\hline $50 / \mathrm{F}$ & H. malignancy & E. faecium & - & - & - \\
\hline $49 / \mathrm{F}$ & Steroid treatment & A. baumannii & A. baumannii & - & - \\
\hline $58 / \mathrm{M}$ & H. malignancy & - & - & - & Aspergillus spp. \\
\hline $53 / \mathrm{M}$ & Solid tumor & P. aeruginosa & P. aeruginosa & - & - \\
\hline
\end{tabular}

H. malignancy = Hematological malignancy; MSSA= methicillin-sensitive Staphylococcus aureus; A. baumannii = Acinetobacter baumannii; S. pneumoniae = Streptococcus pneumoniae; C. pauculus = Cupriavidus pauculus; E. faecium = Entereococcus faecium; $P$. aeruginosa = Pseudomonas aeruginosa.

Finally, in a prior study conducted in our hospital, the performance characteristics of ETA and mini-BAL were compared in 31 patients clinically suspected of having VAP [13]. Mini-BAL demonstrated a greater microorganism isolation rate in patients admitted to the hospital with lower respiratory infection, and a greater recovery of purulent secretions in cases of suspected VAP.

Although studies have demonstrated the usefulness of mini-BAL in VAP, there is insufficient case experience to characterize the utility of the test in immunocompromised hosts. Our study compared the performance of BAL versus mini-BAL in such patients and here we demonstrate a strong correlation between the isolation rates of bacterial and fungal organisms in the two methods: we detected bacteria and/or fungi in $53.1 \%$ of BAL samples and in $56.3 \%$ of mini-BAL samples. On the other hand, our data demonstrate a weak correlation between BAL and ETA for bacterial and/or fungal isolation. The cost of flexible bronchoscopy with BAL was approximately 3-fold higher than the mini-BAL in our study (EUR 125 vs. EUR 40, respectively).

Mini-BAL in Immunocompromised Patients
Table 4. Correlation of the methods

Correlation coefficient

(r)

\begin{tabular}{llr}
\hline BAL bacteriology & mini-BAL bacteriology & 0.850 \\
BAL bacteriology & ETA bacteriology & 0.477 \\
Mini-BAL bacteriology & ETA bacteriology & 0.430 \\
BAL mycology & mini-BAL mycology & 0.821 \\
BAL mycology & ETA mycology & 0.095 \\
Mini-BAL mycology & ETA mycology & -0.176 \\
\hline
\end{tabular}

An evaluation of 249 BAL samples collected from the bronchoscopic examinations of 199 febrile patients with hematological malignancy and pulmonary infiltrates showed the isolation of fungal pathogens in 46 samples [5]. Another study detected fungal pathogens by BAL in 23 of 135 immunocompromised patients with pulmonary infiltrates [8]. In our study, the use of BAL and mini-BAL led to the isolation of Candida albicans in 12 
patients and Aspergillus fumigatus in 1 patient. All 13 patients with fungal pathogens isolated by BAL or miniBAL were considered to have probable fungal pneumonia based on careful consideration of host factors, clinical criteria, and mycological criteria [18]. Although they did not have 'proven fungal disease', antifungal therapy was given to all 13 patients. They were critically ill, mechanically ventilated and immunosuppressed subjects. Furthermore, both direct and indirect mycological evaluations (culture and antigenemia) were positive. Therefore, we had to start immediate treatment with the antifungal agents.

The contribution of the results of diagnostic bronchoscopic methods to survival in immunosuppressed patients is debatable. Prior work demonstrated that the information gained from BAL had no effect on the survival of neutropenic patients with pneumonia [6]. A previous investigation in our hospital showed no relationship between the results of mini-BAL testing and survival rates in patients with VAP [13]. Our current study shows no difference in the rates of survival between patients in whom respiratory pathogens were isolated and those in whom they were not isolated.
The number of subjects in our current study is small due to the highly specific characteristics of this group of immunocompromised patients with bilateral pneumonia who have manifested respiratory failure and a need for invasive mechanical ventilation.

In conclusion, we show a strong correlation between BAL and mini-BAL methods to isolate bacterial and fungal pathogens. Taken in combination, the overall rate of pathogen detection by the two methods in our study was $59.4 \%$, but this high diagnostic rate did not impact survival. Our study suggests that mini-BAL is an efficient, easily applicable, comparable, less-invasive and cost-efficient method, an alternative to BAL for the early diagnostic evaluation of pneumonia in immunocompromised patients with respiratory failure. These data should be validated in a prospective manner at multiple centers with greater numbers of cases.

\section{Acknowledgement}

The authors thank Craig Patterson, MD, for editorial assistance and without whom this study would not have been possible.

\section{References}

-1 Breuer R, Lossos IS, Berkman N, Or R: Pulmonary complications of bone marrow transplantation. Respir Med 1993;87:571579.

-2 Danes C, Gonzalez-Martin J, Pumarola T, Rañó A, Benito N, Torres A, Moreno A, Rovira $\mathrm{M}$, Puig de la Bellacasa J: Pulmonary infiltrates in immunosuppressed patients: analysis of a diagnostic protocol. J Clin $\mathrm{Mi}$ crobiol 2002;40:2134-2140.

$>3$ Ewig S, Glasmacher A, Ulrich B, Wilhelm K, Schäfer H, Nachtsheim KH: Pulmonary infiltrates in neutropenic patients with acute leukemia during chemotherapy: outcome and prognostic factors. Chest 1998;114:444451.

4 Jain P, Sandur S, Meli Y, Arroliga AC, Stoller JK, Mehta AC: Role of flexible bronchoscopy in immunocompromised patients with lung infiltrates. Chest 2004;125:712-722.

5 Hummel M, Rudert S, Hof H, Hehlmann R, Buchheidt D: Diagnostic yield of bronchoscopy with bronchoalveolar lavage in febrile patients with hematologic malignancies and pulmonary infiltrates. Ann Hematol 2008; 87:291-297.
-6 Gruson D, Hilbert G, Valentino R, Vargas F, Chene G, Bebear C, Allery A, Pigneux A, Gbikpi-Benissan G, Cardinaud JP: Utility of fiberoptic bronchoscopy in neutropenic patients admitted to the intensive care unit with pulmonary infiltrates. Crit Care Med 2000;28:2224-2230.

$>7$ Boersma WG, Erjavec Z, van der Werf TS, de Vries-Hosper HG, Gouw AS, Manson WL: Bronchoscopic diagnosis of pulmonary infiltrates in granulocytopenic patients with hematologic malignancies: BAL versus PSB and PBAL. Respir Med 2007;101:317-325.

$>8$ Rañó A, Agustí C, Jimenez P, Angrill J, Benito N, Danés C, González J, Rovira M, Pumarola T, Moreno A, Torres A: Pulmonary infiltrates in non-HIV immunocompromised patients: a diagnostic approach using noninvasive and bronchoscopic procedures. Thorax 2001;56:379-387.

9 Silver MR, Balk RA: Bronchoscopic procedures in the intensive care unit. Crit Care Clin 1995;11:97-109.

10 Turner JS, Willcox PA, Hayhurst MD, Potgieter PD: Fiberoptic bronchoscopy in the intensive care unit - a prospective study of 147 procedures in 107 patients. Crit Care Med 1994;22:259-264.
11 Jin F, Mu D, Chu D, Fu E, Xie Y, Liu T: Severe complications of bronchoscopy. Respiration 2008;76:429-433.

12 Geraci G, Pisello F, Sciumè C, Li Volsi F, Romeo M, Modica G: Complication of flexible fiberoptic bronchoscopy. Literature review. Ann Ital Chir 2007;78:183-192.

13 Bacakoğlu F, Uysal FE, Başoğlu OK, Aydemir Ş, Arda B: The diagnostic value of non-bronchoscopic mini-BAL in ventilator associated pneumonia. Solunum 2007;9: 139-146.

14 Kollef MH, Ward S: The influence of miniBAL cultures on patient outcomes: implications for the antibiotic management of ventilator-associated pneumonia. Chest 1998; 113:412-420

15 Wu CL, Yang DI, Wang NY, Kuo HT, Chen PZ: Quantitative culture of endotracheal aspirates in the diagnosis of ventilator-associated pneumonia in patients with treatment failure. Chest 2002;122:662-668.

16 Rouby JJ, Rossignon MD, Nicolas MH, Martin de Lassale E, Cristin S, Grosset J, Viars P: A prospective study of protected bronchoalveolar lavage in the diagnosis of nosocomial pneumonia. Anesthesiology 1989;71:679685 
17 Mandell LA, Wunderink RG, Anzueto A, Bartlett JG, Campbell GD, Dean NC, Dowell SF, File TM Jr, Musher DM, Niederman MS, Torres A, Whitney CG, Infectious Diseases Society of America, American Thoracic Society: Infectious Diseases Society of America/American Thoracic Society consensus guidelines on the management of community-acquired pneumonia in adults. Clin Infect Dis 2007;44(suppl 2):S27-S72.

18 De Pauw B, Walsh TJ, Donnelly JP, Stevens DA, Edwards JE, Calandra T, Pappas PG, Maertens J, Lortholary O, Kauffman CA, Denning DW, Patterson TF, Maschmeyer G, Bille J, Dismukes WE, Herbrecht R, Hope WW, Kibbler CC, Kullberg BJ, Marr KA, Muñoz P, Odds FC, Perfect JR, Restrepo A, Ruhnke M, Segal BH, Sobel JD, Sorrell TC,
Viscoli C, Wingard JR, Zaoutis T, Bennett JE, European Organization for Research and Treatment of Cancer/Invasive Fungal Infections Cooperative Group, National Institute of Allergy and Infectious Diseases Mycoses Study Group (EORTC/MSG) Consensus Group: Revised definitions of invasive fungal disease from the European Organization for Research and Treatment of Cancer/Invasive Fungal Infections Cooperative Group and the National Institute of Allergy and Infectious Diseases Mycoses Study Group (EORTC/MSG) Consensus Group. Clin Infect Dis 2008;46:1813-1821.

19 Clinical and Laboratory Standards Institute: Performance Standards for Antimicrobial Susceptibility Testing, 15th Information Supplement, Document M100-A15. Wayne, CLSI, 2005
20 Forslöw U, Remberger M, Nordlander A, Mattsson J: The clinical importance of bronchoalveolar lavage in allogeneic SCT patients with pneumonia. Bone Marrow Transplant 2010;45:945-950.

21 Arora SC, Mudaliar YM, Lee C, Mitchell D, Iredell J, Lazarus R: Non-bronchoscopic bronchoalveolar lavage in the microbiological diagnosis of pneumonia in mechanically ventilated patients. Anaesth Intensive Care 2002;30:11-20.

22 Ost DE, Hall CS, Joseph G, Ginocchio C, Condon S, Kao E, LaRusso M, Itzla R, Fein AM: Decision analysis of antibiotic and diagnostic strategies in ventilator-associated pneumonia. Am J Respir Crit Care Med 2003;168:1060-1067. 